\title{
Linfoma de Burkitt asociado a Virus de la Inmunodeficiencia Humana. Reporte de un caso clínico.
}

\section{Burkitt lymphoma associated with Human Immunodeficiency Virus. A clinical case report.}

\section{Coral Torres ${ }^{1}$, Josefina Santana1 ${ }^{1}$ Rodrigo Bravo², Luis Córdova², Ximena Rodríguez³ ${ }^{3}$ Marcelo Mardones²}

1. Cirujano Dentista, Residente Estadía

Perfeccionamiento Cirugía Máxilo Facial Hospital

San José, Santiago, Chile.

2. Cirujano Máxilo Facial Hospital San José, Santiago, Chile. Profesor asistente, Facultad de

Odontología, Universidad de Chile, Santiago, Chile. 3. Médico Cirujano, Anátomo Patólogo Hospital San José, Santiago, Chile.

* Correspondencia autor: Coral Torres | Dirección: San José 1176, Independencia | Teléfono: 225568000 | E-mail: torresm.coral@gmail.com Trabajo recibido el 11/12/2018

Aprobado para su publicación el 05/08/2019.

\section{RESUMEN}

El linfoma de Burkitt es un linfoma de células B maduras altamente agresivo, de baja incidencia. Según la Organización Mundial de la Salud presenta tres variantes: endémico, esporádico y asociado al Virus de la Inmunodeficiencia Humana. Este último representa el $30-40 \%$ de los linfomas no Hodgkin en pacientes seropositivos para virus de la inmunodeficiencia humana. El tratamiento con quimioterapia en combinación a terapia antirretroviral de gran actividad da muy buenos resultados. Se reporta caso de paciente seropositivo para virus de la inmunodeficiencia humana con linfoma de Burkitt en la cavidad oral, tratado con la terapia anteriormente mencionada.

\section{PALABRAS CLAVE:}

Linfoma de Burkitt; Virus de la inmunodeficiencia humana; Terapia de drogas; Terapia antirretroviral; Altamente activa.

Rev. Clin. Periodoncia Implantol. Rehabil. Oral Vol. 12(3); 148-150, 2019.

\section{ABSTRACT}

Burkitt lymphoma is a highly aggressive mature B-cell lymphoma with a low incidence. According to the World Health Organization, it has three variants: endemic, sporadic and associated with Human Immunodeficiency Virus (HIV). The last one represents $30-40 \%$ of non-Hodgkin's lymphomas in HIV seropositive patients. Treatment with chemotherapy in combination with highly active antiretroviral therapy gives very good results. Here is the case report of a HIV seropositive patient who developed Burkitt lymphoma in the oral cavity and received this treatment.

KEY WORDS:

Burkitt lymphoma; Human immunodeficiency virus; Drug therapy; Antiretroviral therapy, Highly active.

Rev. Clin. Periodoncia Implantol. Rehabil. Oral Vol. 12(3); 148-150, 2019.

\section{INTRODUCCIÓN}

Los linfomas son neoplasias del sistema linfático que se originan generalmente en los ganglios linfáticos $u$ otro tejido del organismo por células neoplásicas del tejido linfoide. Existen 2 tipos clínicos: Linfoma de Hodgkin (LH) y Linfoma No Hodgkin (LNH). En Chile, la tasa de incidencia estimada de LH es de 1 por 100 mil habitantes. Mientras que la tasa de incidencia de LNH es de 5,6 por cada 100 mil habitantes, siendo este último 5 veces más frecuente que LH. EI LNH corresponde al tumor maligno linfoide más común en todo el mundo. A nivel de las regiones oral y máxilofacial, es el tercer grupo más común de tumores malignos, principalmente del linaje de células $B^{(1)}$. Los $\mathrm{LNH}$ del tipo de células $B$ son las segundas neoplasias más comunes que se desarrollan en pacientes seropositivos para Virus de la Inmunodeficiencia Humana $(\mathrm{VIH})$, siendo sarcoma de Kaposi el primero(2). Clínicamente, a nivel de la cavidad oral, LNH presenta una predilección de afectación de encías y paladar duro, en pacientes $\mathrm{VIH}$ positivos $^{(3)}$. El linfoma de Burkitt (LB) es un linfoma periférico de células B con una tasa de proliferación extremadamente alta y a menudo con presentación extraganglionar ${ }^{(4)}$. Representa menos del $5 \%$ de $\mathrm{LNH}$, con una incidencia en adultos de 2,5 casos por millón de habitantes por año(5). La Organización Mundial de la Salud clasifica y describe tres variantes clínicas de LB: endémico, esporádico, y asociado a inmunodeficiencia( ${ }^{(6)}$. La variante endémica se desarrolla principalmente en niños de África, está vinculada a la malaria y al virus Epstein Barr (VEB), donde la infección por éste está presente aproximadamente en el 95\% de los LB endémicos con localización habitual en mandíbula. EI VEB aumenta la supervivencia de las células tumorales de LB ${ }^{(7)}$. LB esporádico tiene afectación mundial, comprometiendo el área abdominal e ileocecal, donde su asociación con el VEB es tan sólo del 10 al $20 \%{ }^{(3)}$. Por otra parte, LB asociado a inmunodeficiencia, afecta principalmente a pacientes seropositivos para $\mathrm{VIH}$, y menos del $40 \%$ de los casos en Europa y Estados Unidos está asociado al VEB ${ }^{(2,6)}$. LB representa el $30-40 \%$ de los $\mathrm{LNH}$ en pacientes VIH positivos, pudiendo ser el primer signo de inmunosupresión subyacente. Por lo general los pacientes que padecen esta condición presentan un recuento de células $T C^{(4)}$ superior a 200 por $\mu \mathrm{L}$, es decir en la primera etapa de progresión de la infección por $\mathrm{VIH}^{(6)}$. La infección por VIH provoca una activación policlonal descontrolada de las células $\mathrm{B}^{(2)}$. Genéticamente está asociado a translocaciones resultantes de la sobreexpresión del oncogen cMYC, lo que impulsa la proliferación neoplásica y anormal de células de linfoma de Burkitt ${ }^{(6,8)}$.

\section{INFORME DEL CASO}

Paciente, género masculino de 54 años de edad, $\mathrm{VIH}$ positivo sin tratamiento, hospitalizado en el Hospital San José de Santiago, Chile, por lesión tumoral maxilar. Se indica evaluación por el equipo de Cirugía 
Máxilofacial. Paciente refiere lesión de crecimiento rápido, asintomática, sin asociación a factor etiológico específico, acude a evaluación al hospital debido a gran crecimiento de la lesión. Al examen clínico extraoral se observa importante aumento de volumen de bordes difusos en región premaxilar y nasogeniana, con invasión del piso de las fosas nasales. Piel no se encuentra comprometida clínicamente por la lesión (fig. 1). Al examen ganglionar de la región de cabeza y cuello, se logran pesquisar a la palpación, adenopatía inflamatoria de las regiones submandibular y supraclavicular. En el examen intraoral se evidencia lesión tumoral de consistencia renitente, localizada en reborde alveolar anterosuperior, paladar duro y vestíbulo oral, cubriendo parcialmente superficies oclusales de dientes posterosuperiores. Mucosa que recubre la lesión tumoral presenta zonas con lesiones ulceradas y necróticas (fig. 2). Posterior a la evaluación clínica, se realiza estudio imagenológico completo del paciente, mediante tomografía computarizada (TC), con el objetivo de buscar otras lesiones tumorales que no se evidenciaron en el examen clínico. A nivel de TC de órbitas máxilofacial y senos paranasales, no se observa compromiso lítico. Se descartan alteraciones en TC con contraste de cuello y partes blandas, tórax y cerebro. El equipo planteó diagnóstico clínico de linfoma, y diagnóstico diferencial de carcinoma espinocelular. Adicionalmente se decide realizar, bajo anestesia general, biopsia incisional de la lesión, para confirmación diagnóstica. Procedimiento quirúrgico se realiza tomando muestras en las zonas más representativas de la lesión, sin considerar las zonas ulceradas y/o necróticas. Al estudio histopatológico se observa neoplasia maligna, difusa, constituida por células de tamaño predominantemente grandes, de núcleos redondeados, con leve pleomorfismo y mitosis frecuentes. Se aprecia un patrón clásico de "cielo estrellado", un fenómeno que es causado por la presencia de macrófagos dentro del tejido tumoral (fig. 3). Características histológicas compatibles con diagnóstico de Linfoma de alto grado de estirpe $\mathrm{B}$, concordante con Linfoma de Burkitt atípico asociado a VIH. Posteriormente se realiza un estudio inmunohistoquímico para la confirmación anatomo-morfológica de la muestra, dando positivo para antígeno CD20, CD10, bcl6, MUM1, c-myc y al Factor de proliferación (Ki-67), y negativo para CD5, bcl2, CD30 e hibridación in situ (Cish) EBER, confirmando así el diagnóstico ya mencionado (fig. 4 y 5). Ante el resultado del examen practicado, el paciente es tratado por los equipos de oncología e infectología del Hospital San José. El tratamiento consistió en administrar quimioterapia (QT) asociada a terapia antirretroviral de gran actividad (TARGA). Transcurridas 3 semanas de tratamiento, la lesión tumoral oral remite considerablemente de tamaño (fig. 6). Como hallazgo secundario, posterior al tratamiento médico realizado, se observa en la cavidad oral, candidiasis pseudomembranosa como reacción adversa a los medicamentos utilizados durante la QT y TARGA, la cual fue tratada por antimicótico, con evolución favorable (fig. 7).
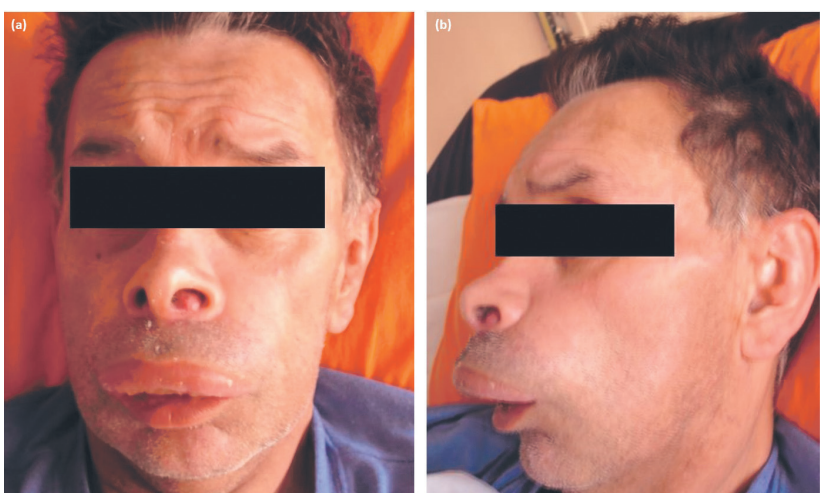

Figura 1. (a) Foto clínica extraoral frontal. (b) Foto clínica extraoral de perfil.

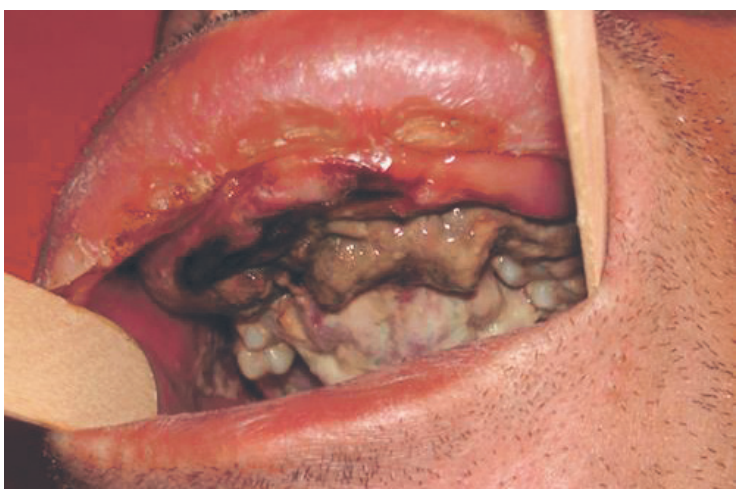

Figura 2. Lesión intraoral.
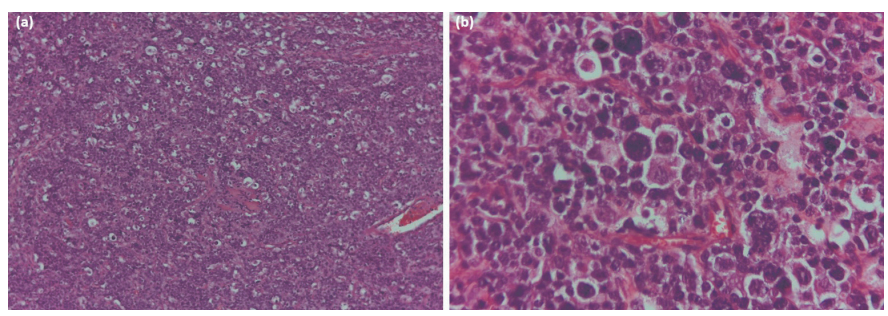

Figura 3. Estudio histopatológico, tinción hematoxilina/eosina. (a) Apariencia de cielo estrellado, aumento 10X. (b) Presencia de células neoplásicas y cuerpos apoptóticos, aumento 40X.
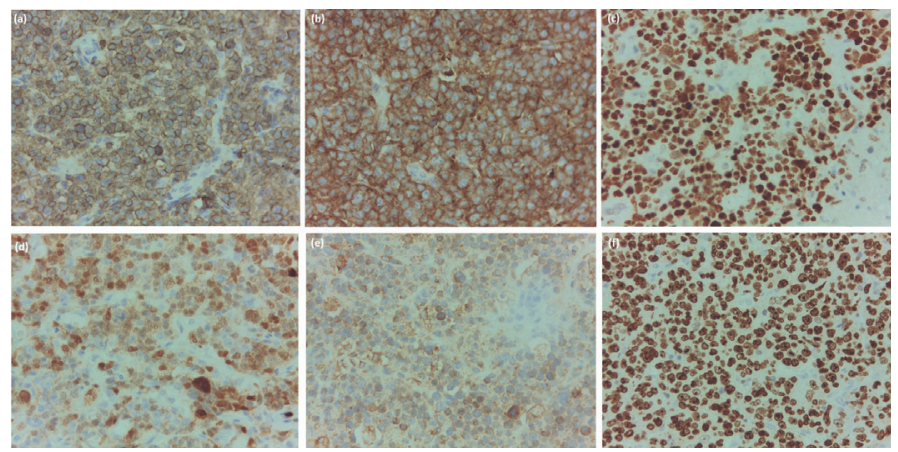

Figura 4. Estudio inmunohistoquímico, contratinción con hematoxilina, aumento 40X, resultados positivos. Positividad para (a) CD20, (b) CD10, (c) bcl-6, (d) MUM-1, (e) c-myc, (f) Ki-67, proliferación positiva para células neoplásicas en más de un $95 \%$.
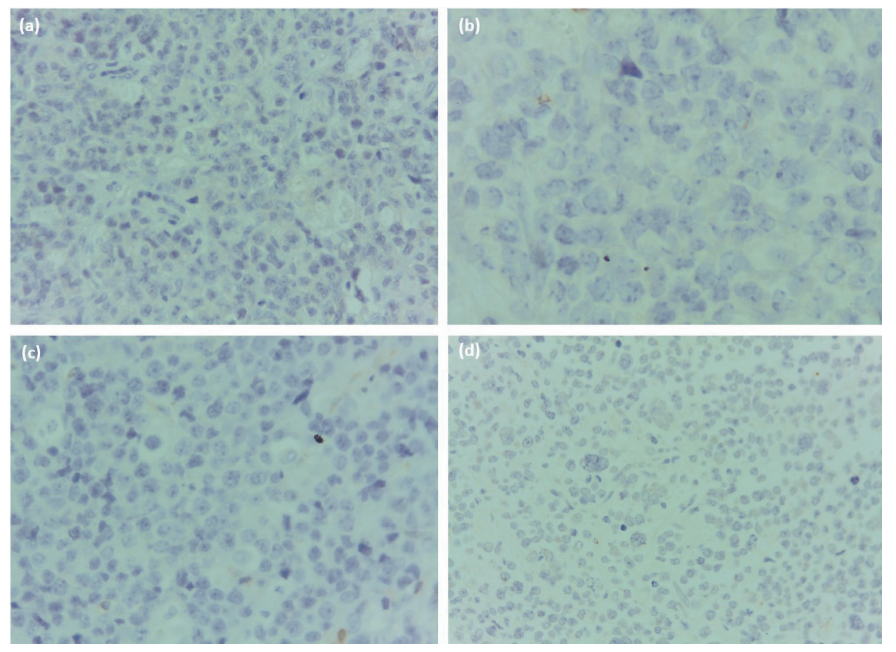

Figura 5. Estudio inmunohistoquímico, contratinción con hematoxilina, aumento 40X, resultados negativosNegatividad para (a) CD30, (b) CD5 (c) blc-2 (d) Hibridación in situ (Cish): EBER

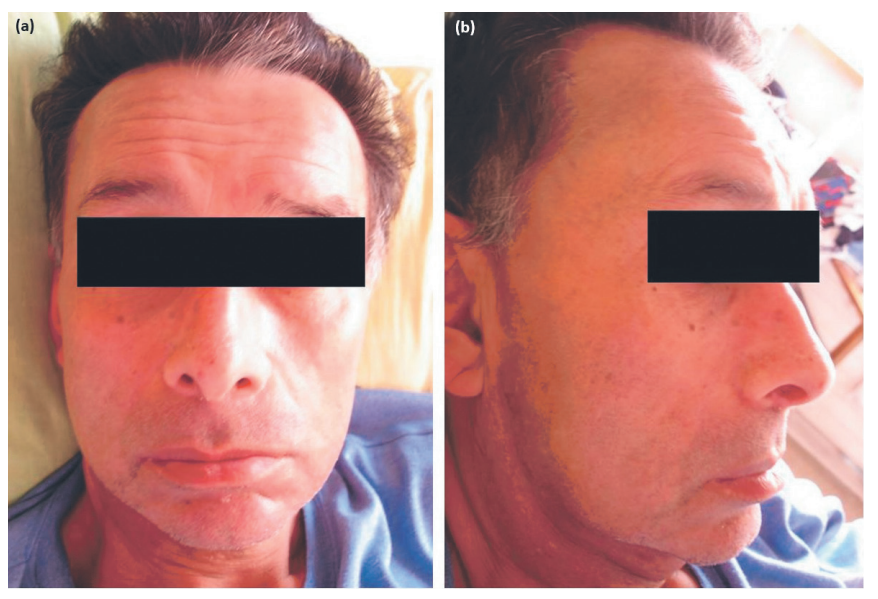

Figura 6. Remisión de lesión posterior a tratamiento con TARGA+QT (A) Foto clínica extraoral frontal. (B) Foto clínica extraoral de perfil 


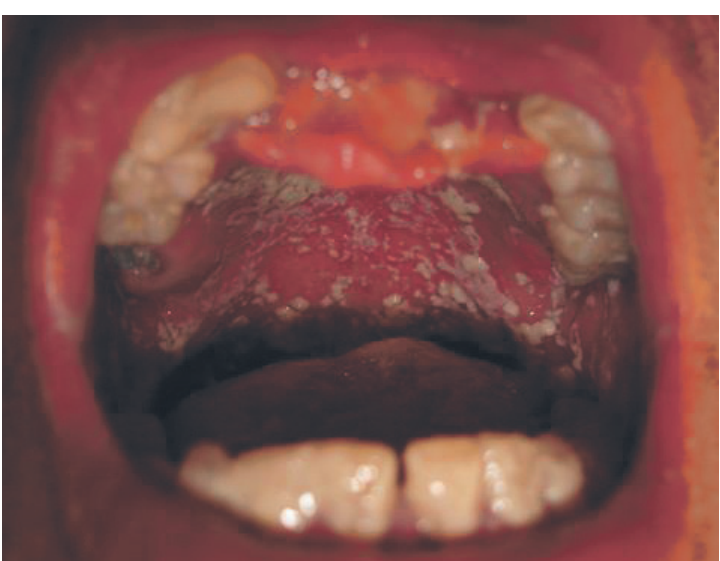

Figura 7. Candidiasis pseudomembranosa posterior a tratamiento con TARGA+QT, foto clínica intraoral.

\section{DISCUSIÓN}

Los pacientes con infección por VIH tienen al menos 50 veces más probabilidades de padecer linfoma. El $30-40 \%$ de estos linfomas corresponde a LB, esto se traduce en un riesgo individual de por vida del $10-20 \%$ de LB para una persona infectada con $\mathrm{VIH}^{(9)}$. Un $40 \%$ de $\mathrm{LNH}$ tiene presentación extranodal, siendo la cavidad oral una localización poco frecuente de los $\mathrm{LNH}$ asociados a $\mathrm{VIH}$, correspondiendo a un 3-4\% de los casos como sitio primario de la neoplasia ${ }^{(10)}$. El caso clínico presentado es un Linfoma de Burkitt en un paciente adulto de 54 años, con presentación extranodal primaria en la cavidad oral, etapa I según clasificación Ann Arbor $^{(8)}$, sin afectación de otros órganos, en paciente con $\mathrm{VIH}$, lo que se traduce en uno de los pocos casos reportados en la literatura. LB se manifiesta clínicamente como una masa de tejido blando con o sin ulceración, necrosis tisular y localizado comúnmente en encía, paladar y mucosa alveolar, con rápido crecimiento y destrucción de tejido circundante ${ }^{(2)}$. Estas características están presentes en nuestro caso, pero se descarta un compromiso lítico del maxilar. El análisis histopatológico da un resultado determinante con respecto al diagnóstico del paciente. Se observan células neoplásicas grandes con escaso citoplasma, núcleos ovalados con cromatina grumosa y abundante apoptosis, lo que coincide con el estudio de Molyneux et al. Esto sumado a los resultados del estudio inmunohistoquímico, pudo determinar un diagnóstico precoz y tratamiento oportuno a este linfoma que se clasifica como de alto grado de malignidad6. Históricamente, los pacientes con linfoma asociado a VIH han sido tratados con QT de menor intensidad debido a la preocupación por la morbilidad relacionada con la inmunosupresión. Sin embargo, en la era actual de TARGA, los pacientes VIH positivos con LB deben ser tratados de manera similar a los pacientes no inmunocomprometidos, con buenos resultados ${ }^{(11)}$. A pesar de su comportamiento agresivo, LB es muy quimiosensible, y varios programas quimioterapéuticos multiagentes intensivos han dado como resultado excelentes tasas de remisión y supervivencia a largo plazo(12). Nuestro estudio reporta una remisión rápida de LB intraoral, transcurridas 3 semanas de tratamiento con QT y TARGA. En un estudio de Schommers et al. se comparó la efectividad de protocolos de quimioterapia en pacientes con LB asociado a VIH (LB-VIH) y linfoma difuso de células $B$ grandes asociado a $\mathrm{VIH}$, analizando sus tasas de supervivencia. A los 2 años de iniciado este estudio, se reporta una tasa de supervivencia de pacientes LB-VIH de un $69 \%$ en comparación con un estudio de Gopal et al. que reportó una tasa del $51 \%{ }^{(13,14)}$. Hoffmann et al. ${ }^{(11)}$ demostró que la tasa de fracaso de QT intensiva agresiva en pacientes VIH positivos con LB fue significativamente más baja en comparación con los pacientes tratados con QT menos agresiva ${ }^{(12)}$. Además, los pacientes toleraron el protocolo agresivo razonablemente bien, particularmente cuando TARGA se incorporó al régimen ${ }^{(11)}$. TARGA combinada con $\mathrm{QT}$ para $\mathrm{VIH}$ ha reducido la incidencia de estas neoplasias y ha mejorado significativamente los resultados clínicos para aquellos que desarrollan linfoma y requieren QT. Pueden tratarse con los mismos regímenes estándar de inmunoquimioterapia utilizados en la población inmunocompetente con expectativas similares a un buen resultado clínico ${ }^{(15)}$. El tratamiento combinado de QT con TARGA, da una tasa de respuesta completa, la cual es alta y oscila entre el 44 y el $60 \%(10)$, mejorando el pronóstico en el tratamiento de pacientes $\mathrm{VIH}$ positivos con presencia de LB.

\section{CONCLUSIÓN}

LB asociado a VIH es un linfoma agresivo de células $B$ que requiere una terapia relativamente intensiva. Los resultados de su tratamiento han mejorado significativamente desde el advenimiento de TARGA para la mayoría de los pacientes con replicación viral bien controlada. Estos resultados se traducen en una mejor respuesta inmune y reducción de complicaciones infecciosas. Su expresión clínica en la cavidad oral puede ser una de las primeras manifestaciones en un individuo $\mathrm{VIH}$ positivo, siendo importante incluirlo en el diagnóstico diferencial de las masas tumorales de la cavidad oral de dichos pacientes. El diagnóstico precoz seguido del tratamiento combinado con QT y TARGA mejora el pronóstico de este tipo de pacientes. La importancia de una biopsia temprana, como fue reportado en nuestro caso, determina un diagnóstico definitivo, tratamiento temprano y oportuno, evitando así el avance de la enfermedad y el compromiso vital del paciente.

\section{RELEVANCIA CLÍNICA}

\section{Justificación científica para el estudio}

El linfoma de Burkitt (LB) representa el segundo tipo de neoplasias más prevalentes en pacientes seropositivos al virus de la inmunodeficiencia humana $(\mathrm{VIH})$. Muchas de estas neoplasias se manifiestan de forma primaria en la cavidad oral, siendo importante su diagnóstico oportuno.

\section{Resultados principales}

La manifestación primaria de LB en la cavidad oral en pacientes VIH positivos requiere un diagnóstico oportuno, evaluación multidisciplinaria y un tratamiento integral que combine quimioterapia con terapia antirretroviral de gran actividad.

\section{Consecuencias prácticas}

Proporcionar conocimientos sobre la manifestación clínica oral de LB asociado a $\mathrm{VIH}$, entregar pautas de diagnóstico clínico e histológico, su tratamiento y pronóstico.

\section{CONFLICTO DE INTERÉS Y FUENTE DE FINANCIAMIENTO}

Los autores declaran no poseer conflictos de interés con ninguna fuente de apoyo financiero, ya sea de tipo privada ni corporativa.

\section{BIBLIOGRAFÍA}

1. MINSAL. Guía Clínica AUGE Linfoma en personas de 15 años y más. Serie Guías Clínicas MINSAL, 2013.

2. Pinisetti S, Nalabolu GR, Uvr C, Tadi DP. HIV associated intra-oral Burkitt's lymphoma: a case report. J Clin Diagn Res. 2013;7:3088-9.

3. Corti M, Villafañe M, Bistmans A, Narbaitz M, Gilardi L. Primary extranodal nonhodgkin lymphoma of the head and neck in patients with acquired immunodeficiency syndrome: a clinicopathologic study of 24 patients in a single hospital of infectious diseases in Argentina. Int Arch Otorhinolaryngol. 2014 Jul;18(3):260-5.

4. El-Naggar A, Chan J, Grandis J, Takata T, Slootweg P. WHO Classification of head and neck tumours. 4th Edition. Lyon: International Agency for Research on Cancer; 2017. p.142.

5. Perkins A, Friedber JW. Burkitt lymphoma in adults. Hematology Am Soc Hemato Educ Program. 2008:341-8.

6. Molyneux E, Rochford R, Griffi B, Newton R, Jackson G, Menon G, et al. Burkitt's lymphoma. Lancet. 2012;379:1234-44.

7. Shannon-Lowe C, Rickinson AB, Bell Al. Epstein-Barr virus-associated lymphomas. Philos Trans R Soc Lond B Biol Sci. 2017 Oct.;372(1732).

8. Neville B, Damm D, Allen C, Chi A. Oral and Maxillofacial Pathology 4th Edition. Canada: Elsevier; 2016. p. 560-56.
9. Noy A. Controversies in the treatment of Burkitt lymphoma in AIDS. Curr Opin Oncol. 2010;22(5):443-8.

10. Corti M, Villafañe MF, Valerga M, Sforza R, Bistmans A, Narbaitz M. Burkitt's lymphoma of the oral cavity in a female with AIDS. Case reportand literature review. Rev Esp Cir Oral Maxilofac. 2015;37(1):44-47

11. Hoffmann C, Wolf E, Wyen C, et al. AIDS-associated Burkitt or Burkitt like lymphoma: short intensive polychemotherapy is feasible and effective. Leuk lymphoma. 2006;47:1872-80.

12. Casulo C, Friedberg J. Treating Burkitt lymphoma in adults. Curr Hematol Malig Rep. 2015;10(3):266-71.

13. Schommers P, Hentrich M, Hoffmann C, Gillor D, Zoufaly A, Jensen B, et al. Survival of AIDS-related diffuse large B-cell lymphoma, Burkitt lymphoma, and plasmablastic lymphoma in the German HIV lymphoma cohort. Br J Haematol. 2015;168(6):806-10.

14. Gopal S, Patel MR, Yanik EL, Cole SR, Achenbach CJ, Napravnik S, et al. Temporal trends in presentation and survival for HIV-associated lymphoma in the antiretroviral therapy era. J Natl Cancer Inst. 2013;21;105(16):1221-9.

15. Kaplan L. HIV-associated Iymphoma. Best Pract Res Clin Haematol. 2012;25:101-117. 